\title{
Syringic acid inhibits apoptosis pathways via downregulation of p38MAPK and JNK signaling pathways in H9c2 cardiomyocytes following hypoxia/reoxygenation injury
}

\author{
SHOU-KUN DING, LI-XIA WANG, LEI-SHENG GUO, PING LUO, \\ JUAN-JUAN DU, ZONG-LEI ZHAO and GUANG-GONG WANG
}

Department of Cardiology, Henan Provincial People's Hospital, Zhengzhou University, Zhengzhou, Henan 450003, P.R. China

Received June 2, 2016; Accepted April 13, 2017

DOI: $10.3892 / \mathrm{mmr} .2017 .6845$

\begin{abstract}
Syringic acid (SA), a naturally occurring O-methylated trihydroxybenzoic acid monomer extracted from Dendrobium nobile Lindl., has been demonstrated to attenuate renal ischemia-reperfusion (I/R) injury. However, the role of SA in myocardial I/R injury is unclear. The present study aimed to clarify the cardioprotective effect of SA in myocardial I/R injury in vitro and explore the potential molecular mechanisms. In the present study, it was revealed that pretreatment with SA increased the viability and inhibited oxidant stress in H9c2 cardiomyocytes that had suffered hypoxia/reoxygenation (H/R). SA also markedly downregulated B-cell lymphoma 2 (Bcl-2) expression and inhibited the expression of Bcl-2-like protein 4 (Bax) and cleaved caspase-3 in H9c2 cardiomyocytes induced by H/R. In addition, SA significantly alleviated $\mathrm{H} / \mathrm{R}$-induced the phosphorylation of p38 mitogen-activated protein kinase (p38MAPK) and c-Jun N-terminal kinase (JNK) in $\mathrm{H} 9 \mathrm{c} 2$ cardiomyocytes. In conclusion, the present study demonstrated that SA inhibits the apoptosis of H9c2 cardiomyocytes following $\mathrm{H} / \mathrm{R}$ injury via reduced activation of the p38MAPK and JNK signaling pathways. The results support the therapeutic usage of SA in the treatment of myocardial infarction.
\end{abstract}

\section{Introduction}

Myocardial infarction (MI) is a major cause of mortality and disability worldwide (1). Although there have been recent advances in the treatment of MI, the general prognosis is unsatisfactory (2-4). Restoration of blood supply, termed reperfusion,

Correspondence to: Dr Guang-Gong Wang, Department of Cardiology, Henan Provincial People's Hospital, Zhengzhou University, 7 Weiwu Road, Zhengzhou, Henan 450003, P.R. China E-mail: wguanggong@126.com

Key words: myocardial infarction, syringic acid, apoptosis, p38 mitogen-activated protein kinase pathway, c-Jun N-terminal kinase pathways has been used to treat ischemic myocardium and prevent further tissue damage. However, reperfusion following a period of prolonged ischemia can often cause myocardial ischemia-reperfusion (I/R) injury, leading to damage of cardiac tissues (5). The underlying mechanisms behind myocardial I/R injury are associated with a number of factors, including substantial free radical production, intracellular calcium overload, increased inflammation, myocardial necrosis and apoptosis (6). Thus, inhibition of oxidative stress and myocardial apoptosis is beneficial in the treatment of myocardial I/R injury.

Syringic acid (SA), a naturally occurring O-methylated trihydroxybenzoic acid monomer extracted from Dendrobium nobile Lindl., exhibits a variety of biological actions including anti-inflammatory, anti-tumor and anti-oxidant properties (7-9). A recent study demonstrated that SA prevented oxidative stress in 1-arginine-induced acute pancreatitis (9). In addition, SA was revealed to protect against I/R injury. Tokmak et al (10) reported that SA pretreatment in spinal cord $I / R$ reduced oxidative stress and neuronal degeneration. SA also attenuated renal I/R injury (11). However, the role of SA in myocardial I/R injury remains to be elucidated. The present study aimed to clarify the cardioprotective effect of SA from myocardial I/R injury in vitro, and the potential molecular mechanisms were also explored. The results demonstrated that SA inhibited apoptosis signaling in $\mathrm{H} 9 \mathrm{c} 2$ cardiomyocytes via downregulation of p38 mitogen-activated protein kinase (p38MAPK) and c-Jun N-terminal kinase (JNK) signaling pathways following hypoxia/reoxygenation $(\mathrm{H} / \mathrm{R})$ injury.

\section{Materials and methods}

Cell culture and treatment. $\mathrm{H} 9 \mathrm{c} 2$ rat cardiomyocyte cell line was obtained from the American Type Culture Collection (Manassas, VA, USA.). Cells were cultured in Dulbecco's modified Eagle's medium (DMEM; Sigma-Aldrich; Merck KGaA, Darmstadt, Germany) with $10 \%$ (v/v) heat inactivated fetal bovine serum (FBS; Gibco; Thermo Fisher Scientific, Inc., Waltham, MA, USA), $100 \mathrm{U} / \mathrm{ml}$ penicillin and $100 \mu \mathrm{g} / \mathrm{ml}$ streptomycin (Sigma-Aldrich; Merck KGaA) at $37^{\circ} \mathrm{C}$ in a humidified incubator with $5 \% \mathrm{CO}_{2}$.

For treatment, $\mathrm{H} 9 \mathrm{c} 2$ cells at a density of $1 \times 10^{4}$ cells/well were pretreated with various concentrations of SA $(0.1,1$ and 
$10 \mu \mathrm{M}$; Sigma-Aldrich; Merck KGaA) for $24 \mathrm{~h}$. Then, the cultures were introduced into a humidified $\mathrm{N}_{2}$ hypoxic chamber $\left(2 \% \mathrm{O}_{2}\right)$ at $37^{\circ} \mathrm{C}$ for $6 \mathrm{~h}$ and then reoxygenated $5 \mathrm{~h}$ at $37^{\circ} \mathrm{C}$ in $5 \% \mathrm{CO}_{2}\left(95 \% \mathrm{O}_{2}\right)$. Normoxic control cells were incubated at $37^{\circ} \mathrm{C}$ in $5 \% \mathrm{CO}_{2}$.

Cell viability assay. Cell viability was detected using a Cell Counting kit-8 (CCK-8) assay. In brief, following treatment, the medium was removed and replaced with fresh DMEM (100 $\mu \mathrm{l} /$ well). Then, $10 \mu \mathrm{l} \mathrm{CCK-8} \mathrm{solution} \mathrm{(Dojindo} \mathrm{Molecular}$ Technologies, Inc., Kumamoto, Japan) was added to each well, and the microplates were incubated at $37^{\circ} \mathrm{C}$ for $2 \mathrm{~h}$. The absorbance was measured at $490 \mathrm{~nm}$ using a microplate reader (Benchmark; Bio-Rad Laboratories, Inc., Hercules, CA, USA).

Cytotoxicity assay. Cell cytotoxicity was measured by a lactate dehydrogenase (LDH) assay using the Cytotoxicity Detection kit (Invitrogen; Thermo Fisher Scientific, Inc.) according to the manufacturer's protocols. Briefly, H9c2 cells were seeded in 96 -well plates at a density of $1 \times 10^{4}$ cells/well. Following treatment, the medium was collected to measure the LDH activity according to the manufacturer's protocol. The colorimetric compound was measured at $530 \mathrm{~nm}$ using a microplate reader (Benchmark; Bio-Rad Laboratories, Inc.).

Measurement of cellular levels of superoxide dismutase (SOD) and malondialdehyde (MDA). H9c2 cells were seeded in 96 -well plates at a density of $1 \times 10^{4}$ cells/well. Following treatment, the activity of SOD in media was analyzed using a SOD kit from Nanjing Jiancheng Bioengineering Institute (Nanjing, China). The MDA level was measured with an MDA kit (Beyotime Institute of Biotechnology, Jiangsu, China). For colorimetric analysis, the absorbance at $532 \mathrm{~nm}$ was recorded using a microplate reader (Spectra Max 190; Molecular Devices, LLC, Sunnyvale, CA, USA).

Western blot analysis. The proteins were extracted from $\mathrm{H} 9 \mathrm{c} 2$ cells using radioimmunoprecipitation lysis buffer (Beyotime Institute of Biotechnology) and the protein concentration was determined by a BCA protein assay kit (Invitrogen; Thermo Fisher Scientific, Inc.). A total of $30 \mu \mathrm{g}$ protein was separated by $10 \%$ SDS-PAGE electrophoresis followed by electroblotting onto a nitrocellulose membrane (GE Healthcare Life Sciences, Little Chalfont, UK). Following blocking with 5\% nonfat dry milk in PBS for $4 \mathrm{~h}$, the membrane was incubated overnight at $4^{\circ} \mathrm{C}$ with primary rabbit anti-mouse antibodies (dilution, 1:1,000) targeting B-cell lymphoma 2 (Bcl-2; cat. no. sc-783), Bcl-2-like protein 4 (Bax; cat. no. sc-6236), cleaved caspase-3 (cat.no. sc-98785), phospho (p)-p38MAPK (cat. no. sc-101759), total p38MAPK (cat. no. sc-535), p-JNK (cat. no. sc-135642), total JNK (cat. no. sc-572) and GAPDH (cat. no. sc-25778; all from Santa Cruz Biotechnology, Inc., Santa Cruz, CA, USA). Subsequently, the membrane was washed and incubated with goat anti-rabbit peroxidase-conjugated immunoglobulin $\mathrm{G}$ (cat. no. sc-516087; Santa Cruz Biotechnology, Inc.) diluted 1:3,000 in the blocking buffer for $1 \mathrm{~h}$. Then the blot was washed in TBST buffer (TBS containing $0.1 \%$ Tween-20) and positive bands were visualized using enhanced chemiluminescence reagents (Bio-Rad Laboratories, Inc.). Densitometry was performed using Gel-Pro Analyzer software version 4.0 (Media Cybernetics, Inc., Rockville, MD, USA).

Statistical analysis. Data are presented as the mean \pm standard deviation. Statistical analyses were performed using SPSS software version 13.0 (SPSS, Inc., Chicago, IL, USA). Statistical analysis was carried out using a one-way analysis of variance followed by a Bonferroni post hoc test for multiple comparisons. $\mathrm{P}<0.05$ was considered to indicate a statistically significant difference.

\section{Results}

$S A$ protects $H / R$-induced $H 9 c 2$ cell injury. The protective effects of SA on H9c2 cell cytotoxicity caused by H/R were investigated. The results of the CCK-8 assay demonstrated that, compared with the normoxia group, cell viability was significantly reduced following $\mathrm{H} / \mathrm{R}$ treatment. Pretreatment with SA for 24 h before H9c2 cells suffered with $6 / 5 \mathrm{~h} \mathrm{H} / \mathrm{R}$ injury markedly inhibited $\mathrm{H} 9 \mathrm{c} 2$ cell injury, compared with the H/R group (Fig. 1A).

It was further analyzed whether SA pretreatment influences the cell death of $\mathrm{H} 9 \mathrm{c} 2$ cells. As demonstrated in Fig. 1B, $\mathrm{H} 9 \mathrm{c} 2$ cells subjected to $6 / 5 \mathrm{~h} \mathrm{H} / \mathrm{R}$ injury caused an marked increase of $\mathrm{LDH}$ release. By contrast, pretreatment of cells with SA for 24 h downregulated LDH release compared with the $\mathrm{H} / \mathrm{R}$ group.

SA inhibits oxidant stress in H/R induced H9c2 cells. Oxidative stress is widely reported to serve a critical role in mediating myocardial I/R injury and thus the effect of SA on oxidant stress in $\mathrm{H} 9 \mathrm{c} 2$ cells in response to $\mathrm{H} / \mathrm{R}$ injury was investigated. As demonstrated in Fig. 2A, H/R treatment significantly decreased SOD level in H9c2 cells compared with the normoxia group and this parameter was significantly increased by SA pretreatment. By contrast, pretreatment with SA significantly reduced MDA levels induced by H/R in $\mathrm{H} 9 \mathrm{c} 2$ cells (Fig. 2B).

SA attenuates $H / R$ induced H9c2 cell apoptosis. Oxidative stress is one of the major stimuli involved in the myocardial apoptosis observed during I/R. Thus, the effect of SA on $\mathrm{H} 9 \mathrm{c} 2$ cell apoptosis in response to $\mathrm{H} / \mathrm{R}$ injury was investigated. As demonstrated in Fig. 3, H9c2 cells exposed to $6 / 5 \mathrm{~h}$ $\mathrm{H} / \mathrm{R}$ injury caused a marked increase of Bax expression. By contrast, pretreatment of cells with SA for $24 \mathrm{~h}$ markedly decreased the H/R-induced Bax expression. SA pretreatment significantly increased the expression of Bcl-2 in H9c2 cells and significantly decreased the level of cleaved caspase- 3 following $\mathrm{H} / \mathrm{R}$.

SA inhibits the activation of p38MAPK and JNK signaling pathways in H9c2 cells. Since p38MAPK and JNK are involved in myocardial injury caused by $\mathrm{I} / \mathrm{R}$, western blot analysis was performed to investigate whether SA inhibited the activation of p38MAPK and JNK in H9c2 cells. As demonstrated in Fig. 4, H/R treatment significantly induced the phosphorylation of p38MAPK and JNK, compared with the normoxia group. However, SA significantly alleviated H/R-induced phosphorylation of p38MAPK and JNK in H9c2 cells. 
A



B

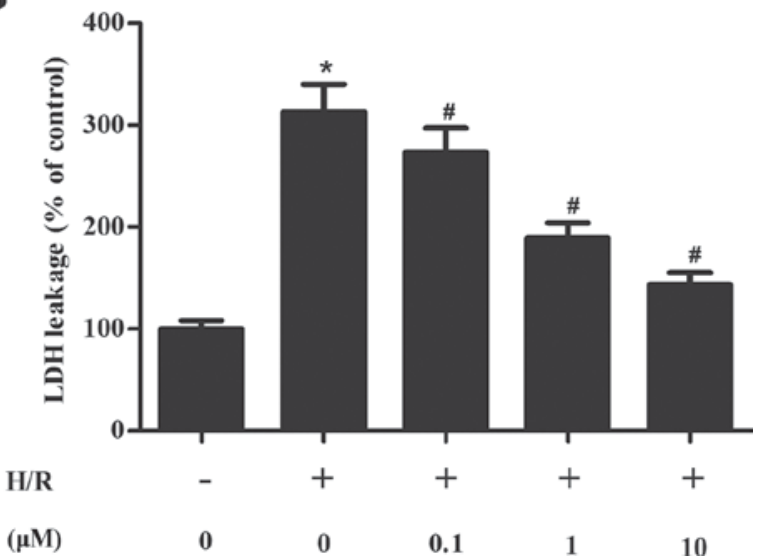

Figure 1. SA protects against H/R-induced H9c2 cell injury. H9c2 cells were pretreated with various concentrations of SA $(0.1,1$ and $10 \mu \mathrm{M})$ for $24 \mathrm{~h}$ and underwent $\mathrm{H} / \mathrm{R}$ injury for $6 / 5 \mathrm{~h}$. (A) Cell viability was detected by the CCK-8 assay. (B) Cell cytotoxicity was assessed by the LDH assay. The data represent the mean \pm standard deviation of three independent experiments. ${ }^{*} \mathrm{P}<0.05$ vs. the normoxia group and ${ }^{\#} \mathrm{P}<0.05$ vs. the $\mathrm{H} / \mathrm{R}$ group. CCK- 8 , Cell Counting kit-8; H/R, hypoxia/reoxygenation; SA, syringic acid; LDH, lactate dehydrogenase.

\section{Discussion}

The present study demonstrated that pretreatment with SA increased the viability and inhibited oxidant stress in H9c2 cardiomyocytes following H/R. SA also markedly upregulated $\mathrm{Bcl}-2$ expression and inhibited increases in Bax and cleaved caspase-3 in H9c2 cardiomyocytes induced by H/R . In addition, SA significantly alleviated the H/R-induced phosphorylation of p38MAPK and JNK in H9c2 cardiomyocytes.

A growing body of evidence indicates that oxidative stress contributes to the development of myocardial I/R injury (12-14). The endogenous defense system, primarily the antioxidant enzyme system (involving SOD and glutathione peroxidase), is critical for attenuating the injury induced by I/R (15). It has been reported that the activity of SOD is considerably reduced following myocardial I/R injury. MDA is the end-product of lipid peroxidization and is increased in myocardial tissue following myocardial I/R injury (16). The present study observed that SA inhibited oxidant stress in H9c2 cardiomyocytes following H/R. Thus, the antioxidant
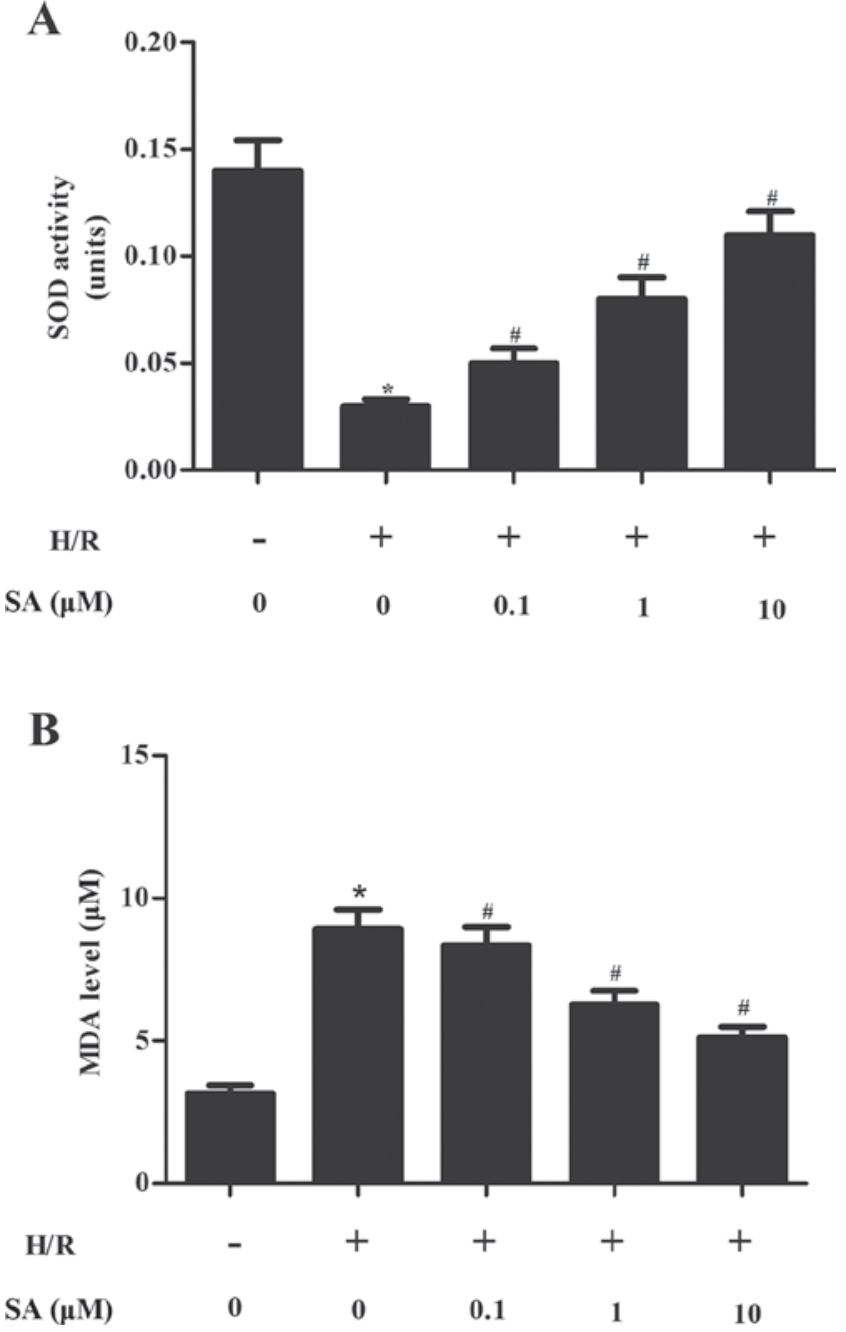

Figure 2. SA inhibits oxidant stress in H/R-induced H9c2 cells. H9c2 cells were pretreated with various concentrations of SA $(0.1,1$ and $10 \mu \mathrm{M})$ for $24 \mathrm{~h}$ and underwent hypoxia/reoxygenation (H/R) injury for $6 / 5 \mathrm{~h}$. (A) the activity of SOD in media was analyzed. (B) MDA level was measured by a method based on a reaction with thiobarbituric acid. The data are presented as the mean \pm standard deviation of three independent experiments. "P<0.05 vs. the normoxia group and ${ }^{\#} \mathrm{P}<0.05$ vs. the H/R group. SOD, superoxide dismutase; $\mathrm{H} / \mathrm{R}$, hypoxia/reoxygenation; SA, syringic acid; MDA, malondialdehyde.

activity of SA may contribute towards a beneficial effect against myocardial I/R injury.

Apoptosis is an active gene-directed cell death process which serves a key role in myocardial reperfusion injury $(17,18)$. Bcl-2 forms a heterodimer with Bax, thereby preventing Bax homodimerization and the activation of caspase-3 (19). Caspase-3, a cysteine protease, also serves a critical role in apoptosis (20). H/R has been demonstrated to elicit cardiomyocyte apoptosis in conjunction with the activation of caspases and an imbalance of pro-/anti-apoptotic proteins (21). Consistent with the previous studies, the present study noted that H/R markedly upregulated expression of Bax and downregulated expression of Bcl-2 in $\mathrm{H} 9 \mathrm{c} 2$ cells. However, pretreatment of cells with SA for $24 \mathrm{~h}$ markedly decreased Bax expression and increased Bcl-2 expression in H9c2 cells induced by H/R. SA pretreatment significantly decreased the level of cleaved caspase- 3 induced by H/R. Thus, the anti-apoptosis activity of SA may contribute towards a beneficial influence against myocardial I/R injury. 
A

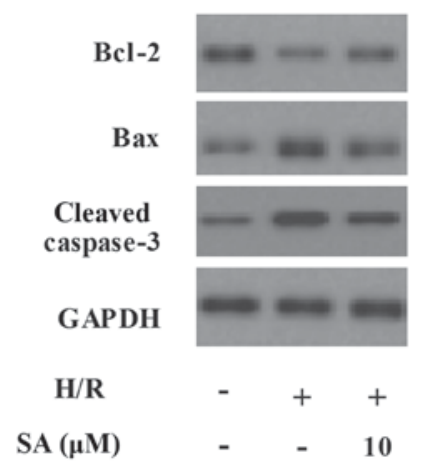

B

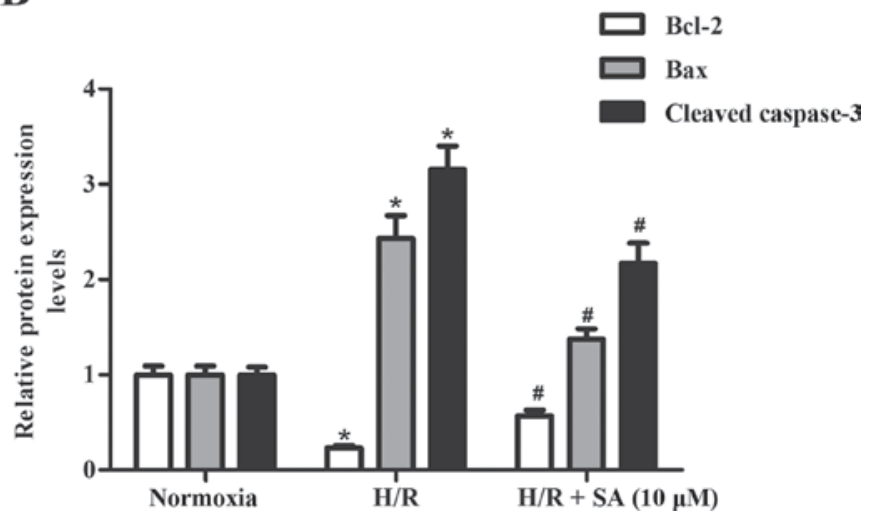

Figure 3. SA attenuates H/R-induced H9c2 cell apoptosis. H9c2 cells were pretreated with $10 \mu \mathrm{M} \mathrm{SA}$ for $24 \mathrm{~h}$ and underwent $\mathrm{H} / \mathrm{R}$ injury for $6 / 5 \mathrm{~h}$. (A) The expression levels of Bcl-2, Bax and cleaved-caspase-3 were detected using western blotting. (B) Band density was measured and normalized to that of GAPDH. The data are presented as the mean \pm standard deviation of three independent experiments. ${ }^{*} \mathrm{P}<0.05$ vs. the normoxia group and ${ }^{\#} \mathrm{P}<0.05$ vs. the H/R group. Bcl-2, B-cell lymphoma 2; Bax, Bcl-2-like protein 4; H/R, hypoxia/reoxygenation; SA, syringic acid.

Previous studies have demonstrated that myocardial I/R is associated with MAPK activation (22-24). Cook et al (25) reported enhanced activation of JNK and p38MAPK in heart tissue from patients with heart failure caused by ischemic disease. Studies have demonstrated that the targeted inhibition of p38MAPK and JNK reduced cardiomyocyte apoptosis and improved cardiac performance following I/R injury (26-28). Engelbrecht et al (29) reported that pre-treatment with SB203580, a p38 inhibitor, produced a significant increase in cell viability and attenuation of the apoptotic index in neonatal cardiomyocytes during simulated $I / R$, while SP600125, a specific JNK inhibitor, caused a significant increase in caspase-3 activation and apoptotic index. Another study confirmed that treatment with the novel JNK inhibitor AS601245 during myocardial ischemia and reperfusion significantly reduced myocardial apoptosis in anaesthetized rats (23). The present study demonstrated that $H / R$ treatment significantly induced the phosphorylation of p38MAPK and JNK. However, SA significantly alleviated H/R-induced phosphorylation of p38MAPK and JNK in H9c2 cells. These results imply that SA can induce cardio-protection via inhibition of p38MAPK and JNK signaling pathways in H9c2 cells.

In conclusion, the present study demonstrated that SA inhibited apoptosis via downregulation of p38MAPK and
A

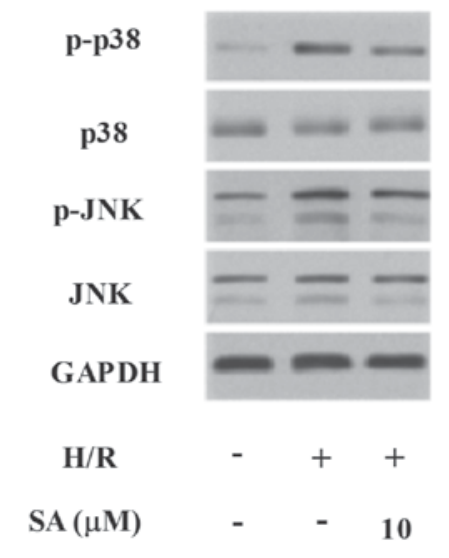

B

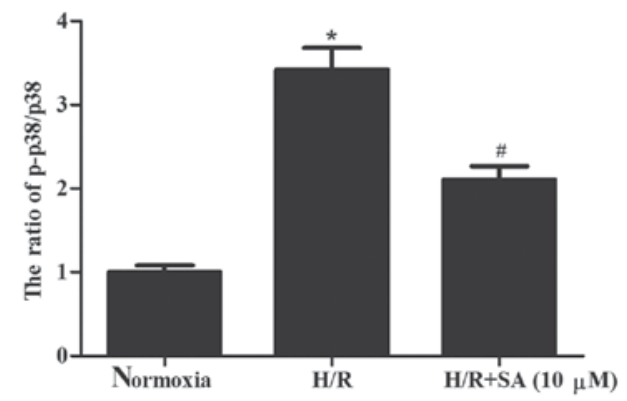

C



Figure 4. SA inhibits the activation of p38MAPK and JNK signaling pathways in $\mathrm{H} 9 \mathrm{c} 2$ cells. $\mathrm{H} 9 \mathrm{c} 2$ cells were pretreated with $10 \mu \mathrm{M}$ SA for $24 \mathrm{~h}$ and underwent $\mathrm{H} / \mathrm{R}$ injury for $6 / 5 \mathrm{~h}$. (A) The expression levels of p-p38, p38, p-JNK and $\mathrm{p}-\mathrm{JNK}$ were detected using western blotting. Band density was measured and normalized to that of GAPDH for (B) p-p38/p38 and (C) p-JNK/JNK. The data represent the mean \pm standard deviation of three independent experiments. " $\mathrm{P}<0.05$ vs. the normoxia group and ${ }^{~} \mathrm{P}<0.05$ vs. the H/R group. $\mathrm{p}-$, phospho-; p38, p38 mitogen-activated protein kinase; JNK, c-Jun N-terminal kinase; H/R, hypoxia/reoxygenation; SA, syringic acid.

JNK signaling pathways in H9c2 cardiomyocytes following $\mathrm{H} / \mathrm{R}$ injury. These results support the therapeutic use of SA in the treatment of MI.

\section{Acknowledgements}

The present study was supported by a project of the Bureau of Public Health of Henan Province (grant no. 201403189). 


\section{References}

1. Hausenloy DJ and Yellon DM: Myocardial ischemia-reperfusion injury: A neglected therapeutic target. J Clin Invest 123: 92-100, 2013.

2. Fintel DJ, Subacius H and Goldberger J: Effect of metoprolol versus carvedilol on survival after myocardial infarction. J Am Coll Cardiol 67: 2091, 2016.

3. Erlinge D, Götberg M, Noc M, Lang I, Holzer M, Clemmensen P, Jensen U, Metzler B, James S and Bøtker HE: Therapeutic hypothermia for the treatment of acute myocardial infarction-combined analysis of the RAPID MI-ICE and the CHILL-MI Trials. Ther Hypothermia Temp Manag 5: 77-84, 2015.

4. Henri O, Houssari M, Pouehe C, Galas L, Nicol L, EdwardsLévy F, Henry JP, Dumesnil A, Schapman D and Thuillez C: Therapeutic lymphangiogenesis improves diastolic function by limiting cardiac edema and fibrosis after myocardial infarction. Circulation 132: A12624-A12624, 2015.

5. Yellon DM and Hausenloy DJ: Myocardial reperfusion injury. New Engl J Med 357: 1121-1135, 2007.

6. Turer AT and Hill JA: Pathogenesis of myocardial ischemia-reperfusion injury and rationale for therapy. Am J Cardiol 106: 360-368, 2010.

7. Ham JR, Lee HI, Choi RY, Sim MO, Seo KI and Lee MK: Anti-steatotic and anti-inflammatory roles of syringic acid in high-fat diet-induced obese mice. Food Funct 7: 689-697, 2016.

8. Abaza MS, Al-Attiyah R, Bhardwaj R, Abbadi G, Koyippally M and Afzal M: Syringic acid from Tamarix aucheriana possesses antimitogenic and chemo-sensitizing activities in human colorectal cancer cells. Pharm Biol 51: 1110-1124, 2013.

9. Cikman O, Soylemez O, Ozkan OF, Kiraz HA, Sayar I, Ademoglu S, Taysi S and Karaayvaz M: Antioxidant activity of syringic acid prevents oxidative stress in l-arginine-induced acute pancreatitis: An experimental study on rats. Int Surg 100: 891-896, 2015

10. Tokmak M, Yuksel Y, Sehitoglu MH, Guven M, Akman T, Aras AB, Cosar M and Abbed KM: The neuroprotective effect of syringic acid on spinal cord ischemia/reperfusion injury in rats. Inflammation 38: 1969-1978, 2015.

11. Sancak EB, Akbas A, Silan C, Cakir DU, Turkon H and Ozkanli SS: Protective effect of syringic acid on kidney ischemia-reperfusion injury. Ren Fail 38: 629-635, 2016.

12. Kanno S, Lee PC, Zhang Y, Ho C, Griffith BP, Shears LL II and Billiar TR: Attenuation of myocardial ischemia/reperfusion injury by superinduction of inducible nitric oxide synthase. Circulation 101: 2742-2748, 2000

13. Tao L, Gao E, Jiao X, Yuan Y, Li S, Christopher TA, Lopez BL, Koch W, Chan L, Goldstein BJ and Ma XL: Adiponectin cardioprotection after myocardial ischemia/reperfusion involves the reduction of oxidative/nitrative stress. Circulation 115 1408-1416, 2007.

14. Lefer DJ and Granger DN: Oxidative stress and cardiac disease. Am J Med 109: 315-323, 2000.

15. Jaeschke $\mathrm{H}$ and Woolbright BL: Current strategies to minimize hepatic ischemia-reperfusion injury by targeting reactive oxygen species. Transplant Rev (Orlando) 26: 103-114, 2012.
16. Cuzzocrea S, Costantino G, Mazzon E, Micali A, De Sarro A and Caputi AP: Beneficial effects of melatonin in a rat model of splanchnic artery occlusion and reperfusion. J Pineal Res 28 . 52-63, 2000.

17. Fliss H and Gattinger D: Apoptosis in ischemic and reperfused rat myocardium. Circ Res 79: 949-956, 1996.

18. Eefting F, Rensing B, Wigman J, Pannekoek WJ, Liu WM, Cramer MJ, Lips DJ and Doevendans PA: Role of apoptosis in reperfusion injury. Cardiovasc Res 61: 414-426, 2004.

19. Burlacu A: Regulation of apoptosis by $\mathrm{Bcl}-2$ family proteins. J Cell Mol Med 7: 249-257, 2003.

20. Porter AG and Jänicke RU: Emerging roles of caspase-3 in apoptosis. Cell Death Differ 6: 99-104, 1999.

21. Wang HC, Zhang HF, Guo WY, Su H, Zhang KR, Li QX, Yan W, Ma XL, Lopez BL and Christopher TA: Hypoxic postconditioning enhances the survival and inhibits apoptosis of cardiomyocytes following reoxygenation: Role of peroxynitrite formation. Apoptosis 11: 1453-1460, 2006.

22. Fülöp N, Zhang Z, Marchase RB and Chatham JC: Glucosamine cardioprotection in perfused rat hearts associated with increased $\mathrm{O}$-linked $\mathrm{N}$-acetylglucosamine protein modification and altered p38 activation. Am J Physiol Heart Circ Physiol 292: H2227-H2236, 2007.

23. Ferrandi C, Ballerio R, Gaillard P, Giachetti C, Carboni S, Vitte PA, Gotteland JP and Cirillo R: Inhibition of c-Jun $\mathrm{N}$-terminal kinase decreases cardiomyocyte apoptosis and infarct size after myocardial ischemia and reperfusion in anaesthetized rats. Brit J Pharmacol 142: 953-960, 2004.

24. Ma XL, Kumar S, Gao F, Louden CS, Lopez BL, Christopher TA, Wang C, Lee JC, Feuerstein GZ and Yue TL: Inhibition of p38 mitogen-activated protein kinase decreases cardiomyocyte apoptosis and improves cardiac function after myocardial ischemia and reperfusion. Circulation 99: 1685-1691, 1999.

25. Cook SA, Sugden PH and Clerk A: Activation of c-Jun N-terminal kinases and p38-mitogen-activated protein kinases in human heart failure secondary to ischaemic heart disease. J Mol Cell Cardiol 31: 1429-1434, 1999.

26. Gao XF, Zhou Y, Wang DY, Lew KS, Richards AM and Wang P: Urocortin-2 suppression of p38-MAPK signaling as an additional mechanism for ischemic cardioprotection. Mol Cell Biochem 398: 135-146, 2015.

27. Zhou L, Zhao D, An H, Zhang H, Jiang C and Yang B: Melatonin prevents lung injury induced by hepatic ischemia-reperfusion through anti-inflammatory and anti-apoptosis effects. Int Immunopharmacol 29: 462-467, 2015.

28. Thomas CJ, Ng DC, Patsikatheodorou N, Limengka Y, Lee MW, Darby IA, Woodman OL and May CN: Cardioprotection from ischaemia-reperfusion injury by a novel flavonol that reduces activation of p38 MAPK. Eur J Pharmacol 658: 160-167, 2011.

29. Engelbrecht AM, Niesler C, Page C and Lochner A: p38 and JNK have distinct regulatory functions on the development of apoptosis during simulated ischaemia and reperfusion in neonatal cardiomyocytes. Basic Res Cardiol 99: 338-350, 2004. 